\title{
Magnetic strip waveguides
}

\author{
Pavel Exner ${ }^{a, b}$, Hynek Kovařík ${ }^{a, c}$ \\ a) Nuclear Physics Institute, Academy of Sciences, $25068 \check{R} e \check{z}$ \\ near Prague, Czechia \\ b) Doppler Institute, Czech Technical University, Břehová 7, \\ 11519 Prague \\ c) Faculty of Mathematics and Physics, Charles University, \\ $V$ Holešovičkách 2,18000 Prague; \\ exner@ujf.cas.cz, kovarik@ujf.cas.cz
}

\begin{abstract}
We analyze the spectrum of the "local" Iwatsuka model, i.e. a twodimensional charged particle interacting with a magnetic field which is homogeneous outside a finite strip and translationally invariant along it. We derive two new sufficient conditions for absolute continuity of the spectrum. We also show that in most cases the number of open spectral gaps of the model is finite. To illustrate these results we investigate numerically the situation when the field is zero in the strip being screened, e.g., by a superconducting mask.
\end{abstract}

\section{Introduction}

There has been a renewed interest recently to problems of magnetic transport in two-dimensional systems. Several papers $\mathrm{MMP}$, FGW1, BP investigated the influence of a weak disorder on current-carrying edge states in a halfplane [Ha, MS, or in a more general domain [FGW2]: roughly speaking, it has been shown that away of the Landau levels the transport survives for both the confining-potential and Dirichlet-boundary border of the halfplane, and that the same is true for Dirichlet regions containing an open wedge. 
On the other hand, it is known for long that transport can be achieved by a suitable variation of the magnetic field alone [Iw if the latter is translationally invariant in one direction. A simple example is represented by the field assuming two different nonzero values in two halfplanes. It has an illustrative semiclassical interpretation in terms of cyclotronic radii CFKS, Sec. 6.5]. On the other hand, the spectrum of this generic Iwatsuka model is purely absolutely continuous, while classically there are many localized states; this shows that the extended character of quantum states plays here an important role.

Furthermore, the transport does not require the existence of different asymptotics; it is possible even if a variation of a constant nonzero magnetic field is restricted to a planar strip, i.e. its transverse profile has a compact support. Two sufficient conditions for absolute continuity of the spectrum are known in this case. In the original paper $[\mathrm{Iw}$ it was shown to be the case if the field derivative has opposite signs to both sides of a given point. Later Mantoiu and Purice MP derived what could be called the "first and third quadrant" condition, i.e. the requirement that $\pm B\left(x_{1}\right) \leq \pm B\left(x_{0}\right) \leq \pm B\left(x_{2}\right)$ holds for some $x_{0}$ and all $x_{1} \leq x_{0} \leq x_{2}$.

In the next section we discuss the "local" Iwatsuka model. For the sake of completeness we describe briefly the procedure of its solution; we skip some details because the subject is discussed thoroughly in [Iw, MP]. We shall derive a pair of new sufficient conditions for the absolute continuity. The first one is a global assumption of the above type: it requires that the field variation is nonzero and does not change sign in the strip under consideration. The other is a rather weak local condition: it is enough that the variation has at least a powerlike growth from zero at the vicinity of the strip edge. The main new ingredient in the second case is a semiclassical estimate of the transverse eigenfunction tails. Notice also that we shall not need the assumption of global field positivity employed in [Iw, MP]. Our conditions strengthen the existing results and represent a step towards the proof of a conjecture put forth in [CFKS, Sec. 6.5] which states that any nonzero (translationally invariant) field variation spreads the Landau levels into a purely absolutely continuous spectrum.

One is also interested, of course, how the spectrum looks as a set. In particular, it is natural to ask whether the spectral bands can overlap, and if so, what is the number of open gaps left. This question is addressed in Section 3. We show there that the number of open gaps is finite provided 
the field variation has a nonzero mean. The case of zero mean is more delicate and we limit ourselves to demonstrating that the conclusion persists under a particular assumption. We believe, however, that the restrictions are rather of a technical nature and we conjecture that the finiteness of open-gap number holds generally for the local Iwatsuka model, up to possible regularity requirements imposed on the field.

The above mentioned mathematical properties of the model represent just one of possible motivations. On the physical side, there has been an interest recently [RPM to the behaviour of an electron gas in a thin film under influence of a perpendicular magnetic field which is screened in a part of the plane by a superconducting "mask". A circular "anti-dot" represents a solvable case which is treated in Ref. [RPM] within several different models.

Suppose now that the mask has the form of a straight strip円. Then we get a particular case of our model, and consequently, the magnetic field creates states which are localized transversally and move along the strip. The form of these states, in particular, their "distance" from the strip is controlled by the velocity; the transverse eigenenergies of fast states are close to the Landau levels of the constant "external" field. In Section 4 we shall investigate numerically two variants of such a system, one with an ideal screening and one with an "overshoot": we will compute the band profiles and illustrate the band overlapping. 1

\section{Local Iwatsuka model}

\subsection{Description of the model}

We consider a charged particle confined to a plane and interacting with a magnetic field perpendicular to the plane. Our basic assumption is that field is translationally invariant in the $y$-direction, nonzero and constant away of

\footnotetext{
${ }^{1} \mathrm{~A}$ negative image situation with a field constant within a strip and zero otherwise was treated in $\mathrm{Ca}$. Here the spectral picture is more complicated: there are transverse eigenvalues of "slow" enough states embedded in the continuum. The same system and several related structures were studied also in $\mathrm{PM}$.

${ }^{2}$ After finishing this paper we learned about recent results of Kim et. al. [KISQ] who studied properties of a nonmagnetic ring. In that case, of course, the spectrum remains to be pure point but one can see a transport analogous to the one discussed here in form of "edge states" moving along the ring.
} 
a strip of a width $2 a$ :

(a) the functional form of the field is

$$
B(x, y)=B(x)=B+b(x)
$$

where $B>0$ and $b$ is bounded and piecewise continuous with $\operatorname{supp} b=$ $[-a, a]$. With an abuse of notation, we employ the same symbol for functions on $\mathbb{R}$ and $\mathbb{R}^{2}$ if they are independent of one variable.

As usual in translationally invariant situations we employ the Landau gauge, i.e. we choose the corresponding vector potential in the form

$$
A_{x}=0, \quad A_{y}(x)=B x+a(x)
$$

with

$$
a(x):=\int_{0}^{x} b(t) \mathrm{d} t
$$

We adopt the natural system of units in which $2 m=\hbar=c=|e|=1$ and assume also that the particle charge equals $-|e|$ having in mind an electron. Then the Hamiltonian $H \equiv H(B, b)$ of our system is

$$
H=(\mathbf{p}+\mathbf{A})^{2}
$$

with the appropriate domain in $L^{2}\left(\mathbb{R}^{2}\right)$.

Since $H$ commutes with $y$-translations, it allows for a standard decomposition. Mimicking the argument of [ [ Iw, Sec. 2] we find that it is unitarily equivalent to the direct integral

$$
\int^{\oplus} H(p) \mathrm{d} p
$$

with the fiber space $L^{2}(\mathbb{R})$ and fiber operator

$$
H(p)=-\partial_{x}^{2}+(p+x B+a(x))^{2}
$$

Since the function $a$ is bounded, the potential is for a fixed $p \in \mathbb{R}$ dominated by the oscillator term, $D(H(p))=D\left(-\partial_{x}^{2}\right) \cap D\left(x^{2}\right)$, the spectrum of $H(p)$ is purely discrete and consists of a sequence of eigenvalues $\epsilon_{n}(p)$ accumulating at $+\infty$ - see [RS, Thm XIII.16].

In the absence of the perturbation $b$ the spectrum consists of the Landau levels, $\left\{(2 n+1) B: n \in \mathbb{N}_{0}\right\}$. We shall first show that the latter belong to the spectrum in the perturbed case too, at least as its accumulation points. 
Lemma 2.1 $\epsilon_{n}(p) \rightarrow(2 n+1) B$ as $|p| \rightarrow \infty$ for any $n \in \mathbb{N}_{0}$.

Proof: We introduce a new variable $z$ and numbers $a^{+}, a^{-}$by

$$
z=x+p / B, \quad a^{ \pm}=\int_{0}^{ \pm a} b(x) \mathrm{d} x .
$$

Then we have $H(p)=-\partial_{z}^{2}+V_{p}(z)$ with $V_{p}(z)=B^{2}\left(z+B^{-1} a(z-p / B)\right)^{2}$. Outside $[p / B-a, p / B+a]$ the potential $V_{p}(z)$ is quadratic and equal to $\left(z+a^{\mp}\right)^{2}$ for $z<(p / B-a)$ and $z>(p / B+a)$, respectively. Thus $V_{p}$ converges for $|p| \rightarrow \infty$ pointwise to the potential of the harmonic oscillator Hamiltonian,

$$
H_{0}=-\partial_{z}^{2}+V_{0}, \quad V_{0}(z)=B z^{2} .
$$

Now we employ a simple trick BEZ]: we check the resolvent convergence on functions $f=\left(H_{0}-\mu\right) \phi$ with $\phi \in C_{0}^{\infty}(\mathbb{R})$. For any $\mu \in \rho(H(p))$ we have

$$
\left\|(H(p)-\mu)^{-1} f-\left(H_{0}-\mu\right)^{-1} f\right\|=\left\|(H(p)-\mu)^{-1}\left(V_{0}-V_{p}\right) \phi\right\| \rightarrow 0
$$

as $|p| \rightarrow \infty$ in view of the compact support. However, such $f$ form a dense set in $L^{2}(\mathbb{R})$, and therefore $H(p) \rightarrow H_{0}$ in the strong resolvent sense and the claim follows by Theorem VIII.14 of [Ka].

\subsection{Absolute continuity}

Next we want to give sufficient conditions under which the spectrum is absolutely continuous. We start with analyticity of the perturbation.

Lemma 2.2 $\{H(p): p \in \mathbb{R} \cup\{\infty\}\}$ is an analytic family of type (A). In particular, each $\epsilon_{n}(\cdot)$ is an analytic function.

Proof: Let us first check the analyticity at infinity. We write

$$
H(p)=H_{0}+a(x)(2 p+2 B x+a(x))
$$

where $H_{0}$ is oscillator Hamiltonian (2.4). Since $a$ is bounded by assumption, we have to check $H_{0}$-boundedness of the second term at the r.h.s. with a suitable relative bound. From the Schwarz inequality we have

$$
\|z f\| \leq\left\|\left(I+z^{2}\right)^{-1} z\right\|\left\|\left(I+z^{2}\right) f\right\| \leq C\left(\left\|z^{2} f\right\|+\|f\|\right)
$$


since $z \mapsto z\left(1+z^{2}\right)^{-1}$ belongs to $L^{2}(\mathbb{R})$. Given $f \in D\left(z^{m}\right)$ denote $f_{\eta}(z):=$ $\eta^{3 / 2} f(\eta z)$; then $\left\|z^{m} f_{\eta}\right\|=\eta^{1-m}\left\|z^{m} f\right\|$. Thus (2.5) acquires the form

$$
\|z f\| \leq C \eta^{-1}\left\|z^{2} f\right\|+C \eta\|f\| ;
$$

in other words for any $\alpha^{\prime}>0$ we can find $\beta^{\prime}>0$ such that

$$
\|z f\| \leq \alpha^{\prime}\left\|z^{2} f\right\|+\beta^{\prime}\|f\| .
$$

On the other hand,

$$
\begin{aligned}
\left\|\left(P^{2}+B^{2} z^{2}\right) f\right\|^{2} & =\left(\left(P^{4}+B^{4} z^{4}+2 B^{2} P z^{2} P+B^{2}\left[P,\left[P, z^{2}\right]\right]\right) f, f\right) \\
& =\left\|P^{2} f\right\|^{2}+B^{4}\left\|z^{2} f\right\|^{2}+2 B^{2}\|z P f\|^{2}-2 B^{2}\|f\|^{2}
\end{aligned}
$$

with $P:=-i \partial_{x}$ holds for all $f \in \mathcal{S}(\mathbb{R})$, which yields the following inequality:

$$
\left\|z^{2} f\right\| \leq B^{-2}\left\|\left(P^{2}+B^{2} z^{2}\right) f\right\|+\sqrt{2} B^{-1}\|f\| .
$$

Combining (2.7) and (2.8) we arrive at

$$
\|z f\| \leq \alpha\left\|\left(P^{2}+B^{2} z^{2}\right) f\right\|+\beta\|f\|
$$

where $\alpha$ can be made arbitrarily small and $\beta=\beta^{\prime}+\alpha^{\prime} B^{-1} \sqrt{2}$. To check the analyticity at any point $p$ we employ the inequalities

$$
\begin{aligned}
\left\|H_{0} f\right\| & \leq\|H(p) f\|+\|a(\cdot)(2 p+2 B \cdot+a(\cdot)) f(\cdot)\| \\
& \leq\|H(p) f\|+2 B\|a\|_{\infty}\|z f\|+\|a\|_{\infty}^{2}\|f\| .
\end{aligned}
$$

Applying (2.6) to the second term we get

$$
\left(1-2 \alpha^{\prime} B\|a\|_{\infty}\right)\left\|H_{0} f\right\| \leq\|H(p) f\|+\left(2 \beta^{\prime} B+\|a\|_{\infty}\right)\|a\|_{\infty}\|f\|,
$$

and since $\alpha^{\prime}$ can be made arbitrarily small, the sought $H(p)$-boundedness of $H\left(p^{\prime}\right)-H(p)$ follows from (2.9). To conclude the proof, it is sufficient to apply [Ka], Thms VII.2.6 and VII.1.8.

Let $\psi_{n}(\cdot, p)$ denote the eigenfunctions of the operator (2.2), i.e.

$$
H(p) \psi_{n}(x, p)=\epsilon_{n}(p) \psi_{n}(x, p) .
$$

Recall that without loss of generality $\psi_{n}$ can be chosen real-valued. Following IW we put

$$
Q_{n, p}(x)=(p+B x+a(x))^{2}-\epsilon_{n}(p)
$$


so $(2.10)$ reads $\psi_{n}^{\prime \prime}(x, p)=Q_{n, p}(x) \psi_{n}(x, p)$, and

$$
l_{n}(x, p)=\psi_{n}^{\prime}(x, p)^{2}-Q_{n, p} \psi_{n}(x, p)^{2} .
$$

Lemma $2.3\left(\llbracket \underline{\mathrm{I}}\right.$, Lemma 3.4]) $l_{n}(x, p) \rightarrow 0$ as $|x| \rightarrow \infty$.

Finally, we have to know that the function

$$
f_{n}(x, p):=(p+x B+a(x)) \psi_{n}(x, p)^{2}
$$

which determines the potential part of the energy form decays fast enough for large $p$.

Lemma 2.4 For any $p$ large enough there is $c(p)>0$ such that

$$
5 c(p) \mathrm{e}^{-p\left(x-x_{0}\right)} \geq f_{n}(x, p) \geq \frac{c(p)}{7} \mathrm{e}^{-3 p\left(x-x_{0}\right)}
$$

holds for all $-a \leq x_{0} \leq x \leq a$.

Proof: By Lemma 2.1 there is $p_{0}>0$ such that $Q_{n, p}(x)>0$ holds for all $x \in[-a, a]$ and $|p|>p_{0}$. More than that, $Q_{n, p}(x)$ grows for a fixed $x$ as $|p| \rightarrow \infty$, which makes it possible to to employ a semiclassical form for the tails of the eigenfunctions: for large enough $p$ we have

$$
\psi_{n}(x, p)=\frac{c_{1}(p)}{Q_{n, p}^{1 / 4}(x)} \exp \left\{-\int_{x_{0}}^{x} \sqrt{Q_{n, p}(\xi)} \mathrm{d} \xi\right\}\left(1+q_{n, p}(x)\right),
$$

where

$$
\left|q_{n, p}(x)\right| \leq \exp \left[\frac{1}{2} \int_{x_{0}}^{x}\left|F_{n, p}^{\prime}\left(x^{\prime}\right)\right| \mathrm{d} x^{\prime}\right]-1
$$

and

$$
F_{n, p}(x)=\int\left\{Q_{n, p}^{-1 / 4}(x) \frac{\mathrm{d}^{2}}{\mathrm{~d} x^{2}}\left(Q_{n, p}^{-1 / 4}(x)\right)\right\} \mathrm{d} x
$$

by [01, Thm VI.2.1]. Substituting for $Q_{n, p}(x)$ from (2.11), we get

$$
\begin{aligned}
F_{n, p}^{\prime}(x) & =\frac{5}{4} \frac{(p+x B+a(x))^{2}(B+b(x))^{2}}{\left[(p+x B+a(x))^{2}-\epsilon_{n}(p)\right]^{5 / 2}} \\
& -\frac{1}{2} \frac{(B+b(x))^{2}+b^{\prime}(x)(p+x B+a(x))}{\left[(p+x B+a(x))^{2}-\epsilon_{n}(p)\right]^{3 / 2}}
\end{aligned}
$$


Thus the integrand in (2.14) can be made arbitrarily small for large enough $p$. Consequently, to a fixed $\lambda>1$ we can always find $p_{\lambda}$ such that

$$
\exp \left[\frac{1}{2} \int_{x_{0}}^{x}\left|F_{n, p}^{\prime}\left(x^{\prime}\right)\right| \mathrm{d} x^{\prime}\right] \leq \lambda .
$$

holds for all $p>p_{\lambda}$. The representation (2.13) is valid at the halfline $x \geq x_{0}$, so the coefficient $c_{1}(p)$ is nonzero; without loss of generality we may suppose that it is positive. The behaviour of $\psi_{n}(x, p) / \psi_{n}\left(x_{0}, p\right)$ is for $x, x_{0} \in[-a, a]$ determined essentially by the exponential factor, because $\left(Q_{n, p}\left(x_{0}\right) / Q_{n, p}(x)\right)^{1 / 2}$ can be then included into the error term. Since $a(\cdot)$ is bounded and we consider $x, x_{0}$ with a limited range, one has

$$
\frac{1}{2} p \leq \sqrt{Q_{n, p}(\xi)} \leq \frac{3}{2} p
$$

for all $p$ larger than some $p_{1}>0$, and therefore

$$
\frac{9 c_{1}(p)^{2}}{2 p} \mathrm{e}^{-p\left(x-x_{0}\right)} \geq \psi_{n}(x, p)^{2} \geq \frac{c_{1}(p)^{2}}{6 p} \mathrm{e}^{-3 p\left(x-x_{0}\right)}
$$

if $p>\max \left(p_{1}, p_{3 / 2}\right)$, where $p_{3 / 2}$ refers to $\lambda=3 / 2$ in (2.15). To conclude the proof, notice that $\lim _{p \rightarrow \infty}(p+x B+a(x)) p^{-1}=1$ holds for $x \in[-a, a]$ and put $c(p):=c_{1}(p)^{2}$.

Remarks 2.5 (i) For the sake of simplicity, we use large enough $p$ in the above claim. However, the assumption (a) is not sensitive to mirror transformation which changes $p$ to $-p$, so the analogous result is valid as $p \rightarrow-\infty$. (ii) We need both an upper and a lower bound, hence we cannot employ $\mathrm{Ag}, \mathrm{He}$ as the authors of Ref. $\mathrm{BP}$ did; we have to resort to a more traditional semiclassical method.

Now we are in position to prove the announced result under one of the following additional assumptions:

(b) $b(\cdot)$ is nonzero and does not change sign in $[-a, a]$,

(c) let $a_{\ell}<a_{r}$, where we have put $a_{\ell}:=\sup \{x: b(x)=0$ in $(-\infty, x)\}$ and $a_{r}:=\inf \{x: b(x)=0$ in $(x, \infty)\}$. There exist $c_{0}, \delta>0$ and $m \in \mathbb{N}$ such that one of the following conditions holds:

$$
|b(x)| \geq c_{0}\left(x-a_{\ell}\right)^{m}, \quad x \in\left[a_{\ell}, a_{\ell}+\delta\right)
$$


or

$$
|b(x)| \geq c_{0}\left(a_{r}-x\right)^{m}, \quad x \in\left(a_{r}-\delta, a_{r}\right] .
$$

Theorem 2.6 Assume (a) and (b), or (a) and (c); then $\left|\epsilon_{n}^{\prime}(p)\right|>0$ for each $n \in \mathbb{N}_{0}$ and all $|p|$ large enough. In particular, the spectrum of $H$ is absolutely continuous.

Proof: To prove the absolute continuity, it is sufficient by Thm XIII.86 of [RS to show that $\epsilon_{n}(\cdot)$ is not constant for any $n \in \mathbb{N}_{0}$. The Feynman-Hellman formula implies

$$
\epsilon_{n}^{\prime}(p)=\left(\psi_{n}(x, p), \frac{\mathrm{d} H(p)}{\mathrm{d} p} \psi_{n}(x, p)\right)=2 \int_{-\infty}^{\infty}(p+B x+a(x)) \psi_{n}(x, p)^{2} \mathrm{~d} x
$$

Let us first investigate the integral on the semi-infinite intervals $(-\infty,-a]$ and $[a, \infty)$. Since

$$
l_{n}^{\prime}(x, p)=-2(B+b(x))(p+B x+a(x)) \psi_{n}(x, p)^{2}
$$

and $b(x)=0$ for $|x|>a$, we can write

$$
\begin{aligned}
& 2 \int_{(-\infty,-a] \cup[a, \infty)}(p+B x+a(x)) \psi_{n}(x, p)^{2} \mathrm{~d} x \\
& \quad=-\frac{1}{B} \int_{(-\infty,-a] \cup[a, \infty)} l_{n}^{\prime}(x, p) \mathrm{d} x=\frac{1}{B}\left[l_{n}(a, p)-l_{n}(-a, p)\right]
\end{aligned}
$$

where we have employed Lemma 2.3. Using (2.19) for the second time, we can rewrite the last expression further as

$$
-\frac{2}{B} \int_{-a}^{a}(B+b(x))(p+B x+a(x)) \psi_{n}(x, p)^{2} \mathrm{~d} x
$$

and thus Eq.(2.2) acquires the form

$$
\begin{aligned}
\epsilon_{n}^{\prime}(p) & =-\frac{2}{B} \int_{-a}^{a} b(x)(p+B x+a(x)) \psi_{n}(x, p)^{2} \mathrm{~d} x \\
& =-\frac{2}{B} \int_{-a}^{a} b(x) f_{n}(x, p) \mathrm{d} x
\end{aligned}
$$


The first claim of the theorem follows immediately since $f_{n}(x, p)$ has a definite sign in $[-a, a]$ for $|p|$ large enough.

Assume now that $(c)$ is valid. We shall consider the condition (2.16) and suppose that $b(x)>0$ in $\left(a_{\ell}, a_{\ell}+\delta\right)$; the other cases can be treated in the same way (cf. Remark 2.5). In view of Lemma 2.4 the r.h.s. of (2.20) can be estimated as follows:

$$
\begin{aligned}
\int_{a_{\ell}}^{a_{\ell}+\delta} & b(x) f_{n}(x, p) \mathrm{d} x+\int_{a_{\ell}+\delta}^{a_{r}} b(x) f_{n}(x, p) \mathrm{d} x \\
\quad \geq & \frac{c(p)}{7} \int_{a_{\ell}}^{a_{\ell}+\delta} b(x) \mathrm{e}^{-3 p\left(x-a_{\ell}\right)} \mathrm{d} x-5 c(p) \int_{a_{\ell}+\delta}^{a_{r}}|b(x)| \mathrm{e}^{-p\left(x-a_{\ell}\right)} \mathrm{d} x \\
\quad \geq & \frac{1}{7} c_{0} c(p) \int_{0}^{\delta} \xi^{m} \mathrm{e}^{-3 p \xi} \mathrm{d} \xi-10 a c_{0} c(p)\|b\|_{\infty} \mathrm{e}^{-p \delta} .
\end{aligned}
$$

Estimating the exponential function in the first integral at the r.h.s. from below by $\max \{0,1-3 p \xi\}$ we get

$$
\epsilon_{n}^{\prime}(p)<-\frac{2 c_{0} c(p)}{B}\left\{\frac{(3 p)^{-m-1}}{7(m+1)(m+2)}-10 a\|b\|_{\infty} \mathrm{e}^{-p \delta}\right\}<0
$$

for all sufficiently large $p$.

Remark 2.7 Since the perturbation is analytic at infinity by Lemma 2.2 we know in fact more than (2.21): under the assumptions of the theorem there are nonzero $c_{ \pm}$and positive integers $m^{( \pm)}$such that

$$
\epsilon_{n}(p)=(2 n+1) B+c_{ \pm} p^{\left.-m^{( \pm}\right)}+\mathcal{O}\left(p^{-m^{( \pm)}-1}\right)
$$

as $p \rightarrow \pm \infty$.

\section{$3 \quad$ Number of gaps}

In addition to the absolute continuity given by Theorem 2.6 we want to know how the spectrum of $H$ looks like as a set. It follows from (2.1) and (2.2) that $\sigma(H)$ consists of a union of spectral bands $I_{n}$ :

$$
I_{n}=\left[\inf _{p \in \mathbb{R}} \epsilon_{n}(p), \sup _{p \in \mathbb{R}} \epsilon_{n}(p)\right] ;
$$

the question is how many gaps between them remain open. In this section we will show that their number is finite because the bands overlap at sufficiently high energies. 


\subsection{Field variation of a nonzero mean}

We shall distinguish two different cases depending on whether the functional $A[b]:=\int_{-a}^{a} b(x) \mathrm{d} x$ vanishes or not. Suppose first that $A[b]$ is nonzero. In that case we have:

Proposition 3.1 Assume $\int_{-a}^{a} b(x) \mathrm{d} x \neq 0$. Let $n(E, p)$ and $n_{0}(E)$ be the numbers of eigenstates of $H(p)$ and $H_{0}$, respectively, with the eigenenergy smaller than $E$. Then for any $m \in \mathbb{N}_{0}$ there exist $p_{0}$ and $E\left(m, p_{0}\right)$ such that

$$
\left(n_{0}(E)-n\left(E, p_{0}\right)\right) \operatorname{sgn} A[b]>m
$$

holds for all $E>E\left(m, p_{0}\right)$.

Proof: The assumption $\int_{-a}^{a} b(x) \mathrm{d} x \neq 0$ is equivalent to $a^{-} \neq a^{+}$. Suppose for definiteness that $A[b]<0$, i.e. $a^{-}>a^{+}$. Since we are interested in the high energy limit we may accept without loss of generality that the field variation support lies in the classically allowed region, $E>\left(p_{0}+x B+a(x)\right)^{2}$ for any $x \in[-a, a]$, and to employ the Bohr-Sommerfeld quantization condition: by [Ti, Thm 7.5] we obtain then

$$
\pi n\left(E, p_{0}\right)=\int_{x^{\ell}(E)}^{x^{r}(E)} \sqrt{E-\left(p_{0}+x B+a(x)\right)^{2}} \mathrm{~d} x+\mathcal{O}\left(E^{0}\right)
$$

where the classical turning points

$$
x^{\ell}(E)=-\frac{\sqrt{E}+p_{0}-a^{-}}{B}, \quad x^{r}(E)=\frac{\sqrt{E}-p_{0}-a^{+}}{B},
$$

satisfy by assumption the inequalities $x^{\ell}(E)<-a$ and $x^{r}(E)>a$.

The idea is to compare (3.1) with the analogous expression for $H_{0}$. Since the spectrum is not affected by a shift of the potential, we change the variable in (2.4) as follows, $z \rightarrow z+a^{-} / B$, and obtain

$$
\pi n_{0}(E)=\int_{x^{\ell}(E)}^{x_{0}^{r}(E)} \sqrt{E-\left(p_{0}+x B+a^{-}\right)^{2}} \mathrm{~d} x+\mathcal{O}(1) .
$$

We have used the fact that by construction the left turning point is the same for both potentials, whereas the right one is moved to

$$
x_{0}^{r}(E)=\frac{\sqrt{E}-p_{0}-a^{-}}{B}
$$


Since $a^{-} \neq a^{+}$we have $a<x_{0}^{r}(E)<x^{r}(E)$. Taking further into account that the two potentials coincide to the left of $-a$, we may write the sought difference as

$$
\begin{aligned}
& \pi\left[n\left(E, p_{0}\right)-n_{0}(E)\right]=\int_{-a}^{a}\left\{\sqrt{E-\left(p_{0}+x B+a(x)\right)^{2}}\right. \\
& \left.\quad-\sqrt{E-\left(p_{0}+x B+a^{-}\right)^{2}}\right\} \mathrm{d} x+\int_{a}^{x_{0}^{r}(E)}\left\{\sqrt{E-\left(p_{0}+x B+a^{+}\right)^{2}}\right. \\
& \left.\quad-\sqrt{E-\left(p_{0}+x B+a^{-}\right)^{2}}\right\} \mathrm{d} x+\int_{x_{0}^{r}(E)}^{x^{r}(E)} \sqrt{E-\left(p_{0}+x B+a^{+}\right)^{2}} \mathrm{~d} x \\
& \quad+\mathcal{O}\left(E^{0}\right)
\end{aligned}
$$

the last term being simply a positive number independent of $E$.

In the first term at the r.h.s. of (3.2) we integrate over a fixed interval, hence the result is $\mathcal{O}\left(E^{-1 / 2}\right)$ as $E \rightarrow \infty$ and may be absorbed into the error term. Furthermore, choosing $p_{0} \geq-a^{+}-a B$ we achieve that the integrand in the second term is positive, hence we have

$$
\pi\left[n\left(E, p_{0}\right)-n_{0}(E)\right] \geq \int_{x_{0}^{r}(E)}^{x^{r}(E)} \sqrt{E-\left(p_{0}+x B+a^{+}\right)^{2}} \mathrm{~d} x+\mathcal{O}\left(E^{0}\right) .
$$

It remains to estimate the last integral. Since the function is nonnegative, decreasing and vanishes only if $x=x^{r}(E)$, we take any $\delta \in\left(0, x^{r}(E)-x_{0}^{r}(E)\right)$ and use a simple bound,

$$
\begin{aligned}
\int_{x_{0}^{r}(E)}^{x^{r}(E)} \sqrt{E-\left(p_{0}+x B+a^{+}\right)^{2}} \mathrm{~d} x & >\int_{x_{0}^{r}(E)}^{x^{r}(E)-\delta} \sqrt{E-\left(p_{0}+x B+a^{+}\right)^{2}} \mathrm{~d} x \\
& \geq \sqrt{2 B \delta \sqrt{E}-B^{2} \delta^{2}}\left(\frac{a^{-}-a^{+}}{B}-\delta\right),
\end{aligned}
$$

which yields the sought result for $E$ large enough. The inequality

$$
n\left(E, p_{0}\right)<n_{0}(E)-m
$$

for $A[b]>0$ is obtained in the same way.

Corollary 3.2 If $A[b] \neq 0$ the number of open gaps in the spectrum of $H$ is finite. 
Proof: Let again $a^{-}>a^{+}$. Since $\epsilon_{n}(p) \rightarrow(2 n+1) B$ as $p \rightarrow \infty$ for a fixed $n \in \mathbb{N}$ by Lemma 2.1, it is sufficient to find $\tilde{n}$ and $\tilde{p}$ such that

$$
\epsilon_{n+1}(\tilde{p})<(2 n+1) B
$$

holds for all $n \geq \tilde{n}$. This follows immediately from Proposition 3.1 with $m=2$. In the opposite case, $a^{-}<a^{+}$, the inequality (3.3) is replaced by $\epsilon_{n-1}(\tilde{p})>(2 n+1) B$.

\subsection{The case of zero mean: an example}

If $A[b]=0$ the situation is more complicated since the two potentials differ only in a subset of the interval $(-a, a)$. We restrict ourselves to an example. As above, put $a_{\ell}:=\sup \{x: b(x)=0$ in $(-\infty, x)\}$, and suppose that there is a number $c \in\left(a_{\ell}, a\right)$ such that $a(x)<0$ holds in $\left(a_{\ell}, c\right)$ and $a(c)=0$.

Let us show that the above conclusion about the finite number of gaps persists in this case. We employ again the Bohr-Sommerfeld condition (3.1) choosing $E$ and $p$ in such a way that $c$ will be the right turning point, $E=(p+B c)^{2}$. It may happen, of course, that there is another classically allowed region to the right of $c$ but changing the potential to $E$ there certainly does not increase the number of bound states, i.e.

$$
\pi n(E, p) \geq \int_{x^{\ell}(E)}^{c} \sqrt{E-(p+x B+a(x))^{2}} \mathrm{~d} x+\mathcal{O}\left(E^{0}\right) .
$$

This has to be compared with the number of oscillator states corresponding to $a=0$. Introducing the variable $y:=c-x$ we get

$$
\begin{aligned}
& \pi\left[n(E, p)-n_{0}(E, p)\right] \geq \int_{0}^{c-a_{\ell}} \sqrt{2 \sqrt{E}-B y+a(c-y)} \sqrt{B y-a(c-y)} \mathrm{d} y \\
&-\int_{0}^{c-a_{\ell}} \sqrt{2 \sqrt{E}-B y} \sqrt{B y} \mathrm{~d} y+\mathcal{O}\left(E^{0}\right) .
\end{aligned}
$$

The difference of the integrands can be written as

$$
\begin{aligned}
& \sqrt[4]{4 E}\left[\sqrt{1-\frac{B y-a(c-y)}{2 \sqrt{E}}} \sqrt{B y-a(c-y)}-\sqrt{1-\frac{B y}{2 \sqrt{E}}} \sqrt{B y}\right] \\
& =\sqrt[4]{4 E}(\sqrt{B y-a(c-y)}-\sqrt{B y})+\mathcal{O}\left(E^{-1 / 4}\right) .
\end{aligned}
$$


The error term can be absorbed into that of (3.2), hence we get

$\pi\left[n(E, p)-n_{0}(E, p)\right] \geq \sqrt[4]{4 E} \int_{0}^{c-a_{\ell}}(\sqrt{B y-a(c-y)}-\sqrt{B y}) \mathrm{d} y+\mathcal{O}\left(E^{0}\right)$,

which gives the desired result since the integral is positive by assumption.

The result can be modified for the other end of $\operatorname{supp} b$, and in a similar vein one can treat cases where $a(x)$ is locally positive close to an endpoint. We will not pursue the line further since we believe that a deeper analysis of the behaviour around the classical turning points would be useful, and at the same time, that the property in question is valid generally:

Conjecture 3.3 The number of open gaps in the local Iwatsuka model is finite for any nonzero $b$ satisfying minimal regularity assumptions such as those formulated in Section. 2.1.

\section{Example: a screened strip}

In this section we shall discuss in more detail the example mentioned in the introduction. Apart of the physical interest mentioned there, the system represents a solvable case which allows us to illustrate the results of the previous sections.

\subsection{The ideal situation}

We shall consider first the simplest situation (without "overshoot" in the terminology of [RPM] when the distance between the film supporting the electrons and the superconducting strip is negligible and the magnetic field is perfectly screened for $|x|<a$. Consequently, the field perturbation $b(x)$ is of the form

$$
b(x)=-B \Theta(|x|-a)
$$

with the vector potential

$$
A_{x}=0, \quad A_{y}(x)=B(x-a) \Theta(x-a)+B(x+a) \Theta(-x-a) .
$$

The fiber operator in the decomposition (2.1) then looks as follows

$$
H(p)=-\partial_{x}^{2}+[p+B(x-a) \Theta(x-a)+B(x+a) \Theta(-x-a)]^{2} .
$$


The real line decomposes into a union of several parts in which the corresponding Schrödinger equation can be solved. Inside the strip the potential term in (4.3) is constant and equal to $p^{2}$, so the eigenfunctions $\psi_{n}(x, p)$ of $H(p)$ are there of the form

$$
\psi_{n}(x, p)=A \exp \left[\kappa_{n}(p) x\right]+B \exp \left[-\kappa_{n}(p) x\right], \quad x \in[-a, a],
$$

where $\kappa_{n}(p):=\sqrt{p^{2}-\epsilon_{n}(p)}$. On the other hand, for $x \notin[-a, a]$ the function $a$ is constant and equal to its boundary values; in view of (2.3) they are

$$
a^{-}=-a^{+}=a B \text {. }
$$

In other words, outside the strip the potential has a parabolic shape and its two branches are shifted mutually by $2 a$. Thus we use the substitutions

$$
z=\left\{\begin{array}{llc}
x+a+p / B & \cdots & x<-a \\
x-a+p / B & \cdots & x>a
\end{array}\right.
$$

denote $r=B z^{2}$ and look for solutions of Eq.(2.10) in the form

$$
\psi_{n}(z, p)=\exp (-r / 2) u_{n}(r, p)
$$

In a usual way we check that $u_{n}(r, p)$ satisfies the confluent hypergeometric equation [AS],

$$
r u_{n}^{\prime \prime}(r, p)+\left(\frac{1}{2}-r\right) u_{n}^{\prime}(r, p)-\alpha u_{n}(r, p)=0
$$

with $\alpha:=\frac{B-\epsilon_{n}(p)}{4 B}$. Consequently, the general solution in the "parabolic regions" is of the form

$$
\psi_{n}(x, p)=\mathrm{e}^{-r / 2}\left\{C_{1} M\left(\alpha, \frac{1}{2}, r\right)+C_{2} U\left(\alpha, \frac{1}{2}, r\right)\right\} .
$$

We should not forget, however, that $r$ in this formula comes from two different substitutions (4.4), and moreover, that the map $z \mapsto r$ is not a bijection. With the exception of the case $p=0$ the real line therefore decomposes into four intervals. In the outer regions the requirement of $L^{2}$ integrability leaves the term with the $U$ function only, while in the middle "parabolic regions" both functions are generally contained in the linear combination. Denote

$$
x_{0}=-\frac{p}{B}-a \operatorname{sgn}(p) .
$$


For $p<0$ this point where the potential reaches zero is to the right of the strip, $a<x_{0}$, and we have

$$
\psi_{n}(x, p)=\left\{\begin{array}{lr}
D_{1} \mathrm{e}^{-r / 2} U(\alpha, \beta, r) & -\infty<x<-a \\
A \exp \left[\kappa_{n}(p) x\right]+B \exp \left[-\kappa_{n}(p) x\right] & -a \leq x \leq a \\
\mathrm{e}^{-r / 2}\left\{C_{1} M(\alpha, \beta, r)+C_{2} U(\alpha, \beta, r)\right\} & a<x<x_{0} \\
D_{2} \mathrm{e}^{-r / 2} U(\alpha, \beta, r) & x_{0} \leq x<\infty
\end{array}\right.
$$

and a similar expression for $p>0$ when $x_{0}<-a$. In the case when $p=0$ we set $x_{0}=-a$ by definition, then $C_{1}=0$ and $C_{2}=D_{1}$. Matching the solutions (4.6) smoothly at the points $-a, a, x_{0}$ we arrive at a homogeneous system of six linear equations for the coefficients $A, B, C_{1}, C_{2}, D_{1}$, and $D_{2}$, which yields an implicit equation for the energy levels $\epsilon_{n}(p)$.

\subsection{The model with an overshoot}

The above model is, of course, idealized because in reality the magnetic field is never fully screened and the vector potential changes sharply near the edges of the screening strip. Another simplified description takes this effect into account by putting

$$
b(x)=-B \Theta(a-|x|)+B|x| \delta(|x|-a),
$$

which corresponds the vector potential

$$
A(x)=x B \Theta(|x|-a) .
$$

Recall that a numerical analysis performed in [RPM] for a screening by a disk suggests that a realistic field profile should lie between these two extrema. The fiber operator in (2.1) now reads

$$
H(p)=-\partial_{x}^{2}+[p+B x \Theta(|x|-a)]^{2} .
$$

Inside the strip we get the same solution as in the model without an overshoot. However, the boundary values of the function $a(x)$ given by (2.3) are now equal to each other,

$$
a^{ \pm}=\lim _{\eta \rightarrow 1+} \int_{0}^{ \pm \eta a} b(x) \mathrm{d} x=0
$$


hence for $x \notin[-a, a]$ we use the same substitutions to the left and to the right of the strip,

$$
z^{\prime}=x+p / B \quad \text { and } \quad r^{\prime}=B\left(z^{\prime}\right)^{2},
$$

In analogy with Sec. 4.1 we find the overshoot solutions $\tilde{\psi}_{n}(x, p)$ of (4.5) in the form

$$
\tilde{\psi}_{n}(x, p)=\left\{\begin{array}{lr}
D_{1}^{\prime} \mathrm{e}^{-r^{\prime} / 2} U\left(\alpha, \beta, r^{\prime}\right) & -\infty<x<-a \\
A^{\prime} \exp \left[\kappa_{n}(p) x\right]+B^{\prime} \exp \left[-\kappa_{n}(p) x\right] & -a \leq x \leq a \\
\mathrm{e}^{-r^{\prime} / 2}\left\{C_{1}^{\prime} M\left(\alpha, \beta, r^{\prime}\right)+C_{2}^{\prime} U\left(\alpha, \beta, r^{\prime}\right)\right\} & a<x<x_{0}^{\prime} \\
D_{2}^{\prime} \mathrm{e}^{-r^{\prime} / 2} U\left(\alpha, \beta, r^{\prime}\right) & x_{0}^{\prime} \leq x<\infty
\end{array}\right.
$$

where $x_{0}^{\prime}=-p / B$. Matching the functions (4.9) smoothly at the points $-a, a, x_{0}$ we arrive again at an implicit equation for the energy levels $\tilde{\epsilon}_{n}(p)$.

\subsection{Numerical results}

Using the Ansätze (4.6) and (4.9) for the two described models, we have solved the matching conditions numerically for several values of the parameters. The obtained band functions, denoted $\epsilon_{n}(p)$ and $\tilde{\epsilon}_{n}(p)$ for the case without and with the overshoot, respectively, are plotted in Fig. 11.

We see that in the ideal situation (without an overshoot) the band functions $\epsilon_{n}(p)$ have the only stationary point at $p=0$. This is true for any $n$ as it follows from Eq. (2.20) which now acquires the form

$$
\epsilon_{n}^{\prime}(p)=2 p \int_{-a}^{a} \psi_{n}(x, p)^{2} \mathrm{~d} x .
$$

The situation is completely different in the presence of an overshoot. Here the fiber operator (4.8) can be rewritten as a sum of $H_{0}$ with the perturbation supported in $[-a, a]$. Consequently, the functions $\tilde{\epsilon}_{n}(p)$ exhibit in general many oscillations corresponding to the harmonic oscillator solutions. Following this argument we would expect that in higher levels these oscillations will be partially "ironed" out for sufficiently large $a$. As an illustration, see the curves for $n=6$ in Figs. 1 and 2 . 

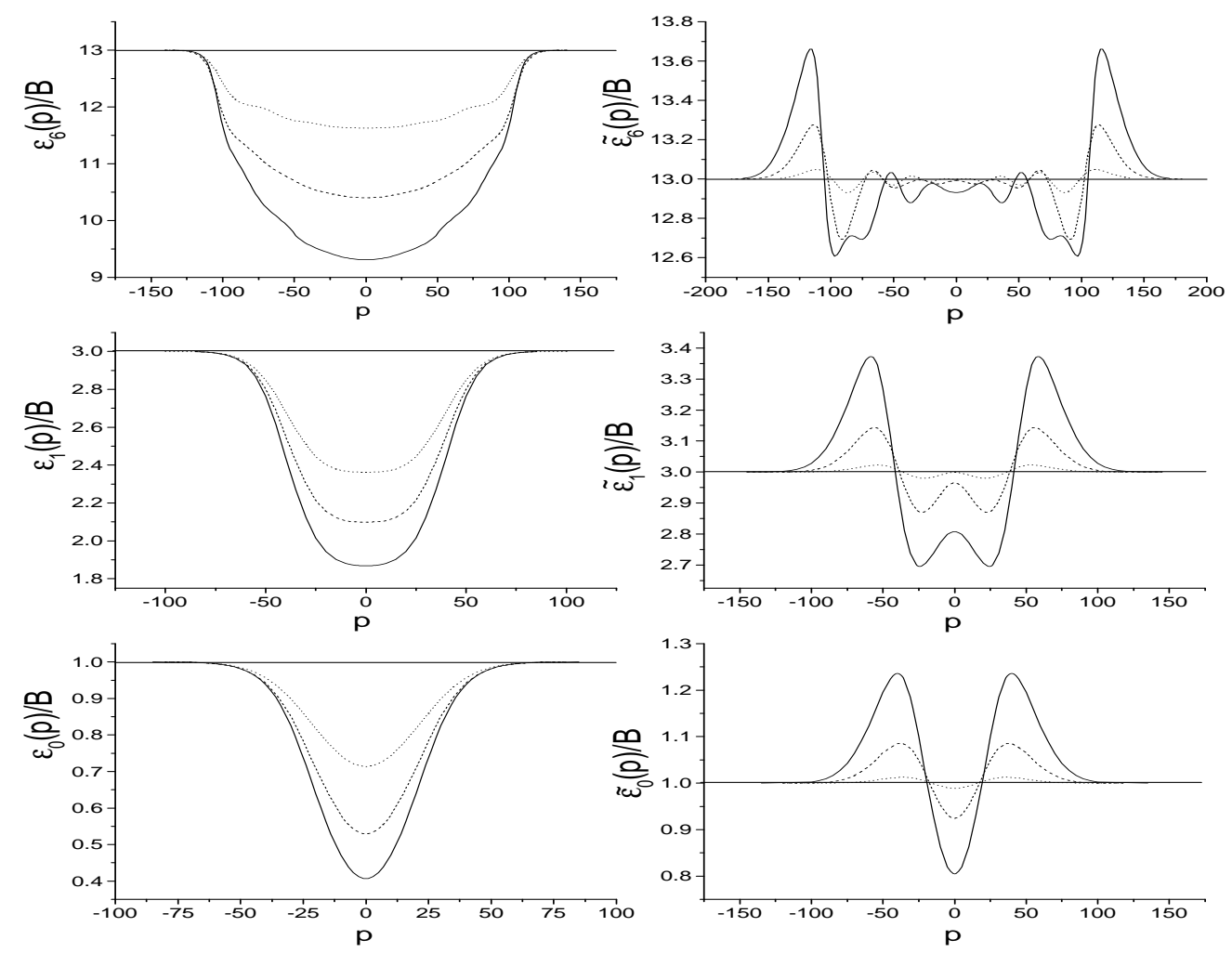

Figure 1: The band functions in the units of $B$ for $B=10^{3}, a=0.01$ (dotted line), $a=0.02$ (dashed line) and $a=0.03$ (full line). (Left) the ideal situation. (Right) the model with an overshoot. The thick lines show the Landau levels.

In Section 3.1 we have shown that if the functional $A[b]$ is nonzero the number of open spectral gaps is finite. Since the field variation (4.1) of the ideal model gives $A[b]=-2 B a$, it follows from Proposition 3.1 that for a large enough band index $n$, depending on $a$ and $B$, the width of the spectral band $I_{n}$ exceeds $2 B$, the distance between Landau levels. Moreover, applying the Bohr-Sommerfeld quantization rule we can easily derive an explicit condition 

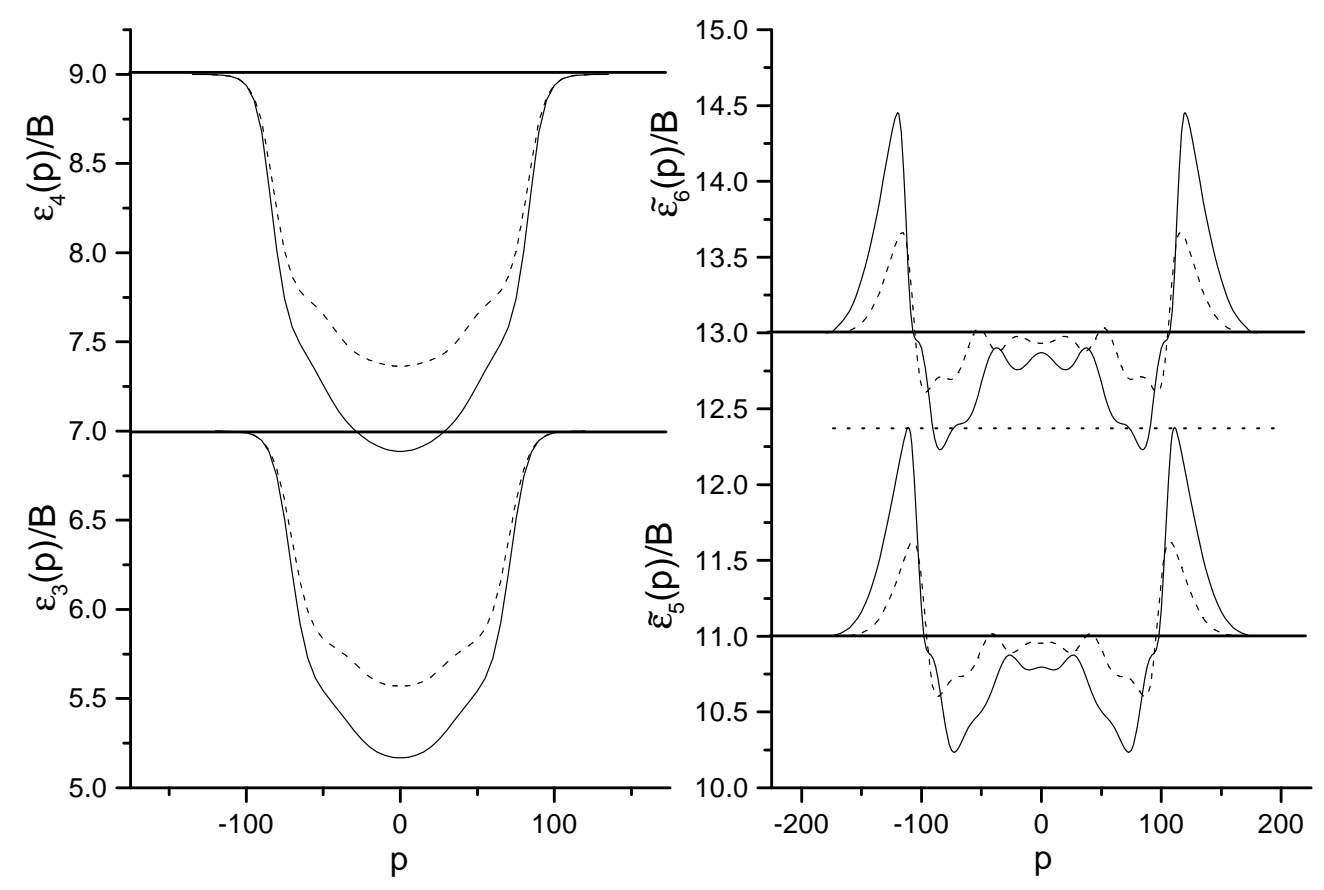

Figure 2: Overlap of the spectral bands. (Left) the ideal situation: $a=0.015$ (dashed line), $a=0.02$ (full line). (Right) the model with an overshoot: $a=0.03$ (dashed line), $a=0.045$ (full line). The field is always $B=10^{3}$.

under which this happens:

$$
n_{\text {overlap }}(a, B) \gtrsim \frac{1}{2}+\frac{\pi^{2}}{8 a^{2} B}
$$

This is shown in Fig 2. In the case of the overshoot model with the field variation given by (4.7) we have no simple condition analogous to (4.11). Nevertheless, the assumptions of the example of Section 3.2 are satisfied here so we know that at sufficiently high energies the spectral bands overlap in this case too.

On a heuristic level, this can be understood from the behavior of the harmonic oscillator solutions. It is well known that for large $n$ they are strongly oscillating but the squared modulus smoothed over a small interval tends as 

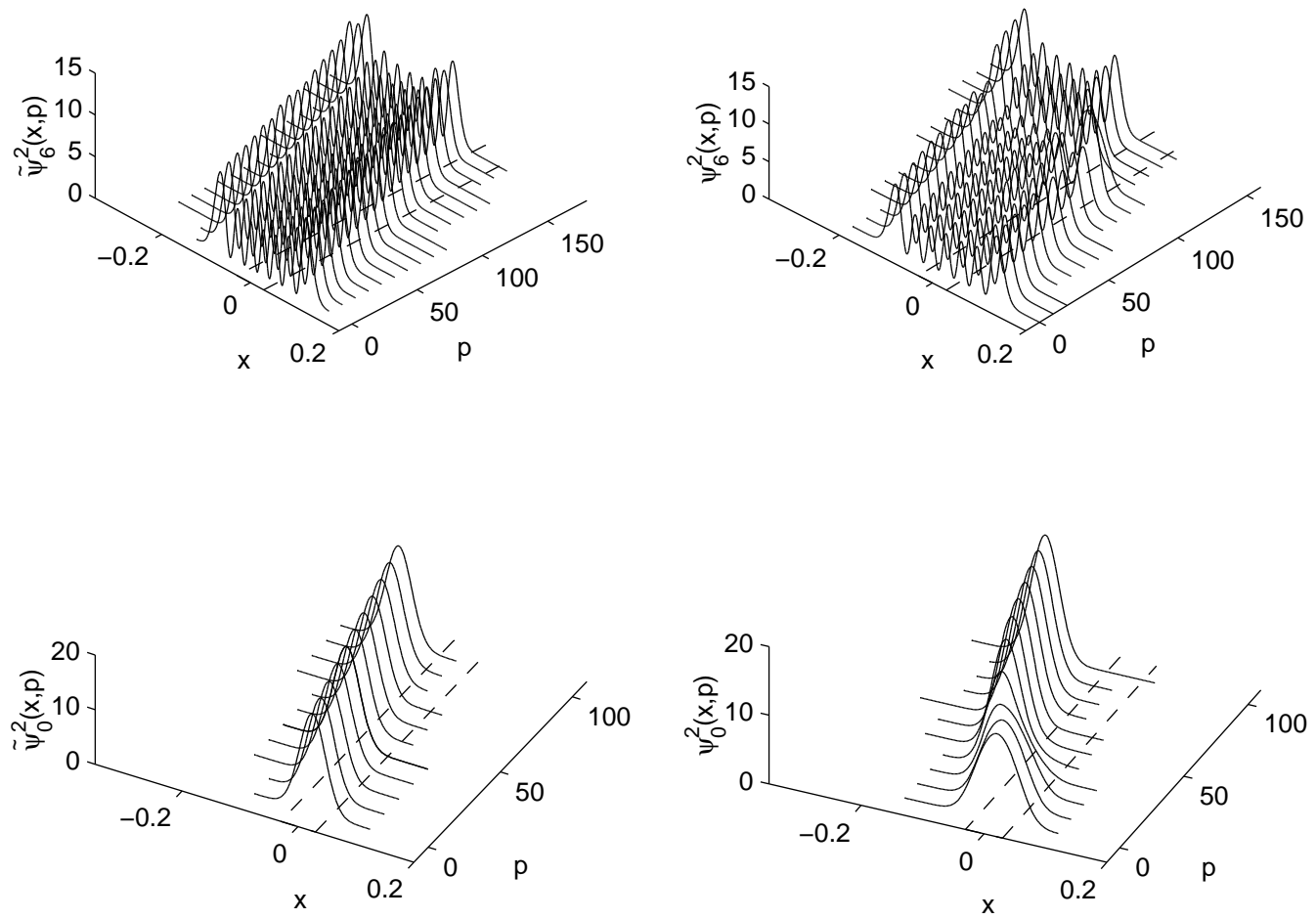

Figure 3: The squared eigenfunctions for $n=0,6$, and different values of $p$. The dashed lines indicate the position of the nonmagnetic strip with $a=0.03$, the field is $B=10^{3}$.

$n \rightarrow \infty$ to the probability density of finding the classical oscillator at a given point [BEH, Section 8.3]. The latter diverges at the turning points. Taking for the difference between $\tilde{\epsilon}_{n}(p)$ and the corresponding Landau level the first-order perturbation theory expression, we expect therefore this quantity to reach its maximum for the values of $p$ corresponding to the vicinity of the turning points. Fig 2 illustrates that it is indeed the case.

Once we have solved the matching conditions for the band functions we can also find the coefficients in (4.6) and (4.9) to obtain complete eigenfunctions $\psi_{n}(x, p)$ and $\tilde{\psi}_{n}(x, p)$, respectively. Fig 3 shows the corresponding probability densities for ground state and the excited state with $n=6$. In the absence of an overshoot it can be seen that as the screened strip $[-a, a]$ 
crosses the center of the distribution, each particular peak of the density becomes broader and more smeared. On the other hand, for large $|p|$ when the "bulk" of the $\left|\psi_{n}(x, p)\right|^{2}$ support lies outside the strip, its shape is close to that of the harmonic-oscillator probability density. In the case of an overshoot the picture is similar but the plot shows a significant deformation for the value of $|p|$ corresponding to the classical turning points.

\section{Acknowledgment}

The research has been partially supported by GA AS under the contract 1048801.

\section{References}

[AS] M. Abramowitz and I.A. Stegun: Handbook of Mathematical Functions, National Bureau of Standards 1964.

[Ag] S. Agmon: Lectures on exponential decay of solutions of second-order elliptic equations, Princeton University Press 1982.

[BEH] J. Blank, P. Exner, M. Havlíček: Hilbert Space Operators in Quantum Physics, AIP Press, New York 1994.

[BEZ] F. Bentosela, P. Exner P., V.A. Zagrebnov: Electron trapping by a current vortex, J.Phys. A: Math. Gen. 31 (1998), L305-311.

[BP] S. De Bièvre, J.V. Pulé: Propagating edge states for a magnetic Hamiltonian, mp-arc 99-78.

[Ca] M. Calvo: Exactly soluble two-dimensional electron gas in magneticfield barrier, Phys. Rev. B48 (1993), 2365-2369.

[CFKS] H.L. Cycon, R.G. Froese, W. Kirsch, B. Simon: Schrödinger Operators with Applications to Quantum Mechanics and Global Geometry, Springer, Berlin 1987.

[FGW1] J. Fröhlich, G.M. Graf, J. Walcher: On the extended nature of edge states of quantum Hall Hamiltonians, math-ph/9903014.

[FGW2] J. Fröhlich, G.M. Graf, J. Walcher: Extended quantum Hall edge states: general domains, mp_arc 99-327.

[Ha] B.I. Halperin: Quantized Hall conductance, current carrying edge states, and the existence of extended states in two-dimensional disordered potential, Phys. Rev. B25 (1982), 2185-2190. 
[He] B. Helffer: Semiclassical Analysis for the Schrödinger Operator and Applications, Lecture Notes in Math., vol. 1336, Springer, Berlin 1966.

[Iw] A. Iwatsuka: Examples of absolutely continuous Schrödinger operators in magnetic fields, Publ. RIMS 21 (1985), 385-401.

[Ka] T. Kato: Perturbation Theory for Linear Operators, Springer, Heidelberg 1966.

[KISC] N. Kim, G. Ihm, H.-S. Sim, K.J. Chang: Electronic structure of a magnetic quantum ring, Phys. Rev. B60 (1999), 8767-8772.

[MS] A.H. MacDonald, P. Středa: Quantized Hall effect and edge currents, Phys. Rev. B29 (1984), 1616-1619.

[MMP] N. Macris, Ph.A. Martin, J.V. Pulé: On edge states in semi-infinite quantum Hall systems, cond-mat/9812367.

[MP] M. Mantoiu, R. Purice: Some propagation properties of the Iwatsuka model, Commun. Math. Phys. 188 (1997), 691-708.

[Ol] F.W.J. Olver: Asymptotics and Special Functions, Academic Press, New York 1974.

[PM] F.M. Peeters, A. Matulis: Quantum structures created by nonhomogeneous magnetic fields, Phys. Rev. B48 (1993), 15166-15174.

[RS] M. Reed and B. Simon: Methods of Modern Mathematical Physics, I. Functional Analysis, IV. Analysis of Operators, Academic Press, New York, 1972, 1978.

[RPM] J. Reijniers, F.M. Peeters, and A. Matulis: Quantum states in a magnetic anti-dot, Phys. Rev. B 59 (1999), 2817-2823.

[Ti] E.C. Titchmarsh: Eigenfunction Expansions, Clarendon Press, Oxford 1949. 